\title{
SPECTRAL THEORY FOR SUBNORMAL OPERATORS BY
}

\author{
R. G. LAUTZENHEISER
}

\begin{abstract}
We give an example of a subnormal operator $T$ such that C $\backslash \sigma(T)$ has an infinite number of components, int $(\sigma(T))$ has two components $U$ and $V$, and $T$ cannot be decomposed with respect to $U$ and $V$. That is, it is impossible to write $T=T_{1} \oplus T_{2}$ with $\sigma\left(T_{1}\right)=\bar{U}$ and $\sigma\left(T_{2}\right)=\bar{V}$. This example shows that Sarason's decomposition theorem cannot be extended to the infinitely-connected case.

We also use Mlak's generalization of Sarason's theorem to prove theorems on the existence of reducing subspaces. For example, if $X$ is a spectral set for $T$ and $K \subset X$, conditions are given which imply that $T$ has a nontrivial reducing subspace $\mathfrak{R}$ such that $\sigma(T \mid \mathscr{T}) \subset K$. In particular, we show that if $T$ is a subnormal operator and if $\Gamma$ is a piecewise $C^{2}$ Jordan closed curve which intersects $\sigma(T)$ in a set of measure zero on $\Gamma$, then $T=T_{1} \oplus T_{2}$ with $\sigma\left(T_{1}\right) \subset \sigma(T) \cap \overline{\operatorname{ext}(\Gamma)}$ and $\sigma\left(T_{2}\right) \subset \sigma(T) \cap \overline{\operatorname{int}(\Gamma)}$.
\end{abstract}

Introduction. In this paper operator will always refer to a bounded linear transformation from a Hilbert space $\mathcal{H}$ into $\mathcal{H}$. An open problem in operator theory which has been the subject of many papers in recent years is the invariant subspace problem. That is, does an arbitrary operator $T$ have a nontrivial invariant subspace? One approach to solving this problem utilizes the spectrum of the operator. For example, if $\sigma(T)$, the spectrum of $T$, is the union of two nonempty disjoint compact sets $K_{1}$ and $K_{2}$, then the Riesz-Dunford functional calculus may be applied to show that $T$ has invariant subspaces $\Re_{i}$ such that $\sigma\left(T \mid \mathscr{T}_{i}\right)=K_{i}$ for $i=1,2$. Since neither set $K_{i}$ is equal to $\sigma(T), \mathfrak{K}_{i}$ must be nontrivial. The Jordan canonical form of a matrix illustrates the success of this approach when the dimension of $\mathcal{H}$ is finite.

This approach suggests the more general question: for which nonempty compact sets $K$ does there exist an invariant subspace $\mathscr{T}$ for $T$ so that $\sigma(T \mid \Re)=K$ ? If $\sigma(T)$ is finite, then the answer is every compact subset of $\sigma(T)$. On the other hand, if $T$ is the unilateral shift, then every nontrivial invariant subspace $\mathfrak{R}$ of $T$ has the property that $\sigma(T \mid \mathfrak{N})=\sigma(T)$. Note that if it is possible to give an affirmative answer for a nonempty set $K$ which is not $\sigma(T)$, then the corresponding subspace $\mathscr{N}$ is necessarily nontrivial.

The purpose of this paper is to investigate the above question when certain conditions are imposed on the operator $T$. We shall consider compact

Received by the editors April 17, 1978 and, in revised form, October 20, 1978.

AMS (MOS) subject classifications (1970). Primary 47A15, 47A25, 47B20; Secondary 47A10. Key words and phrases. Spectral set, subnormal operator, Gleason part, peak set. 
spectral sets $X$ for $T$ which have a nontrivial "algebraic" decomposition. That is, instead of decomposing $X$ topologically, we shall identify $X$ with the maximal ideal space of $R(X)$ and then try to answer the question when $K$ is the closure of a nontrivial Gleason part for $R(X)$. This generalizes a result of $D$. Sarason [27], in which he found conditions so that if $K$ is the closure of a component of int $X$, then the answer is yes. In $\S 3$, we give an example which shows the limitations and, at the same time, leads to a generalization of Sarason's result.

An important class of operators for which the invariant subspace problem remains open consists of the subnormal operators. An operator $T$ is subnormal if it has a normal extension or, equivalently, $T=N \mid \mathfrak{T}$ where $N$ is normal and $\mathfrak{N}$ is an invariant subspace for $N$. Later we shall see that the best examples of operators to which our results apply are those whose spectra are spectral sets. Subnormal operators have this property and are essentially the only known examples to which our results may be applied. We shall be most interested in finding conditions on $K_{1} \cap K_{2}$ so that if $T$ is subnormal and $\sigma(T)=K_{1} \cup K_{2}$, then $T$ has reducing subspaces $\Re_{i}$ such that $\sigma\left(T \mid \mathscr{T}_{i}\right)$ $=K_{i}, i=1,2$. A subspace $\Re$ is reducing for $T$ if $\Re$ is invariant for both $T$ and $T^{*}$ or, equivalently, if $P$ is the projection onto $\mathfrak{T}$, then $P T=T P$. If $\Re$ is reducing for $T$, then $T$ may be written as $T=T_{1} \oplus T_{2}$ where $T_{1}=T \mid \mathscr{T}$ and $T_{2}=T \mid \mathfrak{R}^{\perp}$. In this paper, the notation $T=T_{1} \oplus T_{2}$ will not necessarily imply that $T$ has a nontrivial reducing subspace. For instance, $T_{1}$ might be trivial which means that it is the 0-operator on the 0-dimensional Hilbert space and $T_{2}$ would then be equal to $T$.

2. Spectral sets and Sarason's theorem. If $T$ is an operator on $\mathcal{H}$ and $X$ is a closed set containing $\sigma(T)$, then $X$ is called a spectral set for $T$ if $\|f(T)\|<$ $\|f\|_{X}$ for all rational functions $f$ with poles off $X$. J. von Neumann introduced spectral sets in [32] and proved that the unit disk is a spectral set for all contractions. He also proved that $\sigma(T)$ is equal to the intersection of all spectral sets for $T$. C. Berger [5] has improved this result by showing that the intersection of all compact spectral sets is $\sigma(T)$. In this paper we shall only consider compact spectral sets.

Even though an operator $T$ possesses many compact spectral sets, it is usually quite difficult to exhibit any other than the disk about the origin of radius $\|T\|$. However, under certain conditions, it is possible to show that $\sigma(T)$ is a spectral set for $T$. For instance, using the spectral theorem, it is easy to show the spectrum of a normal operator is a spectral set. In [3], S. K. Berberian noted that $\sigma(T)$ is a spectral set for $T$ if and only if $\|f(T)\|$ is the spectral radius of $f(T)$ for all rational functions $f$ with poles off $\sigma(T)$. This follows directly from the spectral mapping theorem for rational functions. This result and the fact that the spectrum of the minimal normal extension of 
a subnormal operator is contained in the spectrum of the subnormal operator (see Lemma (3.2)) imply that the spectrum of a subnormal is a spectral set. If, however, $T$ is assumed to be only hyponormal $\left(T T^{*}<T^{*} T\right)$, then $\sigma(T)$ need not be a spectral set for $T$ (see Clancey [8] or Wadhwa [33] for an example).

If $X$ is compact, then $R(X)$ will denote the uniform closure of the rational functions with poles off $X$. If $X$ is a spectral set for $T$, then it is possible to associate an operator $f(T)$ with each $f \in R(X)$. That is, if $f \in R(X)$ and $\left\{f_{n}\right\}$ is a sequence of rational functions converging to $f$, then the sequence of operators $\left\{f_{n}(T)\right\}$ has a limit in $\mathscr{B}(\mathcal{H})$, the operators on $\mathcal{H}$, and we denote this limit by $f(T)$. It is also easy to see that $f(X)$ is a spectral set for $f(T)$. In case $X=\sigma(T)$, then $f(\sigma(T))=\sigma(f(T))$ and the correspondence between $f$ and $f(T)$ establishes an isometric isomorphism from $R(X)$ into $\Re(\mathcal{H})$.

The following two well-known results illustrate the connection between spectral sets and reducing subspaces and also give an indication as to the type of results which follow. One theorem involves a topological condition on $X$, and the other, a condition on the algebra $R(X)$.

TheOREM (2.1) (Williams [37]). If $X=X_{1} \cup X_{2}$ is a spectral set for $T$ where $X_{1}$ and $X_{2}$ are disjoint and compact, then $T=T_{1} \oplus T_{2}$ with $\sigma\left(T_{i}\right) \subset X_{i}$, $i=1$, 2. In particular, if $\sigma(T) \cap X_{i} \neq \varnothing$ for $i=1,2$, then $T$ has a nontrivial reducing subspace.

Theorem (2.2) (Von NeumanN [32]). If $X$ is a spectral set for $T$ and $R(X)=C(X)$, then $T$ is normal.

Both theorems may be derived from the following lemma.

LEMMA (2.3) (BERGER [4], FOAIS [12], LEBOW [20]). If $X$ is a spectral set for $T$, then for each $x, y \in \mathcal{H}$, there exists a complex Borel measure $\mu_{x y}$ supported on $\partial X$ such that $\left\|\mu_{x, y}\right\|<\|x\|\|y\|, \mu_{x, x}>0$ with $\left\|\mu_{x, x}\right\|=\|x\|^{2}$, and

$$
(f(T) x, y)=\int f(z) d \mu_{x, y}
$$

for all $f$ in $R(X)$.

Proof. Since $R(X)$ is isometrically isomorphic to $R(X) \mid \partial X$, the map $f \mapsto(f(T) x, y)$ defines a continuous linear functional on $R(X) \mid \partial X$. Continuity follows from the assumption that $X$ is a spectral set for $T$. Extending the map to $C(\partial X)$ by the Hahn-Banach theorem while preserving the norm and then applying the Riesz representation theorem gives the desired measure.

To prove Theorem (2.1), note that $\chi_{X_{i}}$, the characteristic function of $X_{i}$, is in $R(X)$ and then use Lemma (2.3) to show that $\chi_{x_{i}}(T)$ is a projection and that $\sigma\left(T \chi_{X_{i}}(T)\right) \subset X_{i}$. Theorem (2.2) follows by observing that $f(z)=\bar{z}$ is in $R(X)$ and that $f(T)=T^{*}$. That is, 


$$
(f(T) x, x)=\int \bar{z} d \mu_{x, x}=\overline{\int z d \mu_{x, x}}=\left(T^{*} x, x\right) .
$$

Since $f(T)$ commutes with $T, T$ is normal.

In 1965 , D. Sarason proved the following remarkable theorem relating the components of int $X$ to reducing subspaces of $T$.

TheOREM (2.4) (SARASON [27]). Let $X$ be a spectral set for $T$ such that $C \backslash X$ is connected. If $G_{1}, G_{2} \ldots$ are the components of int $X$, then

$$
T=N \oplus\left(\sum_{i=1}^{\infty} \oplus T_{i}\right)
$$

where $N$ is normal with $\sigma(N) \subset \partial X$ and $\bar{G}_{i}$ is a spectral set for $T_{i}$. In particular, $\sigma\left(T_{i}\right) \subset \bar{G}_{i}$.

Sarason's proof depends entirely on the existence of a normal dilation of $T$. That is, if $\mathbf{C} \backslash X$ is connected and $X$ is a spectral set for $T$, then there exists a normal operator $S$ defined on a larger Hilbert space such that $\sigma(S) \subset \partial X$ and $f(T)=P f(S) \mid \mathcal{H}$ for all $f$ in $R(X)$. The operator $P$ is the projection onto $\mathcal{H}$. See Lebow [20] for a proof. Since it is not known whether normal dilations exist even in the simple case when $X$ is an annulus (see Arveson [2]), to generalize Theorem (2.4) to more complicated sets would require different techniques. In $\$ 3$, we give an example to show that it cannot be generalized to include sets $X$ whose complement has an infinite number of components. However, if $\mathbf{C} \backslash X$ has a finite number of components, then the theorem is true. This will be covered in $\$ 4$.

Decomposition theorems such as Theorem (2.4) are quite useful when trying to show that $T$ has nontrivial reducing subspaces. For instance, if $N$ is nontrivial, then $T$ surely has a nontrivial reducing subspace. That is, it is well known that normal operators have an abundance of reducing subspaces. If $N$ is trivial, then to use the decomposition theorem, we must show that at least two of the $T_{i}$ 's are nontrivial. The operator $T_{i}$ will, in fact, be nontrivial if $\sigma(T) \cap G_{i} \neq \varnothing$. To see this, first consider the following lemma.

LeMMA (2.5). If $X_{i}$ is a spectral set for $T_{i} \in \mathscr{B}\left(\mathcal{H}_{i}\right)$ for $i=1,2,3, \ldots$ and $X=\bar{\cup}_{i=1}^{\infty} X_{i}$ is compact, then $X$ is a spectral set for $T=\sum_{i=1}^{\infty} \bigoplus T_{i}$.

Proof. Since $\left\|T_{i}\right\|<\sup \left\{|z| \mid z \in X_{i}\right\}<\sup \{|z||| z \mid \in X\}$, it follows that $T$ is an operator on $\mathcal{H}=\sum_{i=1}^{\infty} \oplus \mathcal{K}_{i}$. If $f$ is a rational function with poles off $X$, then $\left\|f\left(T_{i}\right)\right\|<\|f\|_{X_{i}}<\|f\|_{X}$ for $i=1,2, \ldots$ Therefore $\|f(T)\|=$ $\sup \left\{\left\|f\left(T_{i}\right)\right\|\right\}<\|f\|_{X}$ and $X$ is a spectral set for $T$.

Note that since $X$ is a spectral set for $T, \sigma(T) \subset X$. In the special case that $X_{i}=\sigma\left(T_{i}\right)$, then we have that $\sigma(T)=\bigcup_{i=1}^{\infty} \sigma\left(T_{i}\right)$. In case there are only a finite number of operators, then it is easy to show that $\sigma(T)=\cup_{i=1}^{n} \sigma\left(T_{i}\right)$. 
Proposition (2.6). Let the notation be as in Theorem (2.4). If $\sigma(T) \cap G_{i} \neq$ $\varnothing$, then $T_{i}$ is nontrivial and if $\sigma(T) \notin \overline{\cup_{i=1}^{\infty} G_{i}}$, then $N$ is nontrivial.

Proof. If $T_{i}$ were trivial, then

$$
\sigma(T)=\sigma(N) \cup \sigma\left(\sum_{j \neq i} \oplus T_{j}\right)
$$

But the previous lemma implies

$$
\sigma\left(\sum_{j \neq i} \oplus T_{j}\right) \subset \overline{\bigcup_{j \neq i} G_{j}} .
$$

Therefore, for $\lambda \in \sigma(T) \cap G_{i}$, it follows that $\lambda \in \sigma(N)$ or $\lambda \in \overline{\cup_{j \neq i} G_{j}}$, but this is impossible since $\sigma(N) \subset \partial X$ and $G_{i} \cap \overline{\cup_{j \neq i} G_{j}}=\varnothing$. Thus $T_{i}$ is nontrivial.

If $\sigma(T) \not \subset \bar{\cup}_{i=1}^{\infty} G_{i}$, then by $\operatorname{Lemma}(2.5), \sigma(T) \neq \sigma\left(\sum_{i=1}^{\infty} \oplus T_{i}\right)$. Since

$$
\sigma(T)=\sigma(N) \cup \sigma\left(\sum_{i=1}^{\infty} \bigoplus T_{i}\right),
$$

it follows that $N$ is nontrivial.

If $X$ is the union of two closed disks whose intersection is a point, $X$ is a spectral set for $T$, and $\sigma(T)$ intersects both components of int $X$, then we see that $T$ has a nontrivial decomposition $T=T_{1} \oplus T_{2}$. (The normal operator may be absorbed into the operators $T_{1}$ and $T_{2}$.) When $X$ has this simple form, it is possible to use other techniques to show that $T$ has nontrivial invariant subspaces (see Stampfli [29]). However, if $X$ is a cornucopia (i.e., a disk with a ribbon which winds around and clusters on the boundary of the disk) with the same properties as above, then again it follows that $T$ has a nontrivial decomposition $T=T_{1} \oplus T_{2}$. Other known techniques for producing reducing subspaces seem to fail in this situation. It appears that J. G. Stampfli was the first to observe that Theorem (2.4) may be used in this case.

3. An example. In this section we give an example to show that Theorem (2.4) cannot be extended to the case when $\mathbf{C} \backslash X$ has an infinite number of components. The example is a subnormal operator $T$ satisfying:

(i) $\mathbf{C} \backslash \sigma(T)$ has an infinite number of components,

(ii) int $\sigma(T)$ has two components $U$ and $V$, and

(iii) $T$ cannot be written as $T_{1} \oplus T_{2}$ with $\sigma\left(T_{1}\right) \subset \bar{U}$ and $\sigma\left(T_{2}\right) \subset \bar{V}$.

In fact, $T$ satisfies a somewhat stronger property than (iii): namely, for each nontrivial invariant subspace $\mathfrak{N}$ of $T, \sigma(T) \subset \sigma(T \mid \Re)$. In order to define $T$ and show that it has the desired properties, we need to introduce some terminology and recall a few facts.

If $W$ is an open subset of $\mathbf{C}$ whose boundary is a rectifiable Jordan curve $\Gamma$ and $\varphi$ is a conformal mapping from $\Delta=\{z|| z \mid<1\}$ onto $W$, then $\varphi$ has a 
one-to-one continuous extension to $\bar{\Delta}$ which is absolutely continuous on $\partial \Delta$. If $E$ is a measurable subset of $\Gamma$, then the arc length of $E$ is $\int_{\varphi^{-1}(E)}\left|\varphi^{\prime}\left(e^{i t}\right)\right| d t$. Since $\varphi^{\prime}\left(e^{i t}\right) \neq 0$ a.e. on $\partial \Delta$, it follows that a measurable set $E \subset \Gamma$ has arc length zero if and only if $\varphi^{-1}(E)$ has measure zero. This is standard material and may be found in the books by Duren [11] or Tsuji [30].

The concept of a representing measure will also be needed. If $X$ is compact and $a \in X$, then $m$ is a representing measure for evaluation at $a$ on $R(X)$ if $m$ is a probability measure supported on $\partial X$ and $f(a)=\int f d m$ for all $f \in R(X)$. It is easy to show that each $a \in X$ has a representing measure and if $R(X)$ is a Dirichlet algebra (i.e., $\operatorname{Re}(R(X) \mid \partial X)$ is dense in $\left.C_{R}(\partial X)\right)$, then each $a \in X$ has a unique representing measure. If $\mathbf{C} \backslash X$ is connected, then it is well known that $R(X)$ is a Dirichlet algebra.

LEMMA (3.1). Let $W$ and $\varphi$ be as above, and fix an element $z_{0}=r e^{i \theta}$ in $\Delta$. If $m$ is the unique representing measure for evaluation at $\varphi\left(z_{0}\right)$ on $R(\bar{W})$, then

(i) if $f \in L^{1}(m)$, then $f \circ \varphi \in L^{1}\left(P_{r}(\theta-t) d t / 2 \pi\right)$ and

$$
\int f d m=\int f \circ \varphi\left(e^{i t}\right) P_{r}(\theta-t) d t / 2 \pi
$$

where $P_{r}$ is the Poisson kernel,

(ii) $m$ and arclength on $\Gamma$ are mutually absolutely continuous, and

(iii) ( $F$. and $M$. Riesz theorem on $\Gamma$ ) if $f \in H^{1}(m)=L^{1}(m)$-closure of $R(\bar{W})$, then either $f(z) \neq 0$ a.e. $[m]$ or $f(z)=0$ a.e. $[m]$.

Proof. If $u$ is the continuous image of the measure $P_{r}(\theta-t) d t / 2 \pi$ under $\varphi$, then for $f \in L^{1}(u), f \circ \varphi \in L^{1}\left(P_{r}(\theta-t) d t / 2 \pi\right)$ and

$$
\int f d u=\int(f \circ \varphi)\left(e^{i t}\right) P_{r}(\theta-t) \frac{d t}{2 \pi}
$$

(see [17, p. 180]). For $f \in R(\bar{W}), f \circ \varphi \in R(\bar{\Delta})$ and

$$
\int f d u=\int(f \circ \varphi)\left(e^{i t}\right) P_{r}(\theta-t) \frac{d t}{2 \pi}=(f \circ \varphi)\left(z_{0}\right)=f\left(\varphi\left(z_{0}\right)\right) .
$$

Since $m$ is the unique representing measure for evaluation at $\varphi\left(z_{0}\right), m=u$. Therefore (i) is true. Since $P_{r}(\theta-t) d t / 2 \pi$ and the arc length on $\partial \Delta$ are mutually absolutely continuous and $m$ is the continuous image of $P_{r}(\theta-$ $t) d t / 2 \pi$ under $\varphi$, (ii) also follows.

To prove (iii), suppose $f \in H^{1}(m)$ and $f(z)=0$ for all $z \in E$ where $m(E)>0$. Let $\left\{f_{n}\right\}$ be a sequence in $R(\bar{W})$ converging to $f$ in $L^{1}(m)$. By (i),

$$
\begin{aligned}
\int\left|f_{n}-f\right| d m & =\int\left(\left|f_{n}-f\right| \circ \varphi\right)\left(e^{i t}\right) P_{r}(\theta-t) \frac{d t}{2 \pi} \\
& =\int\left|f_{n} \circ \varphi-f \circ \varphi\right|\left(e^{i t}\right) P_{r}(\theta-t) \frac{d t}{2 \pi} .
\end{aligned}
$$


Therefore, $f \circ \varphi \in \mathcal{H}^{1}=L^{1}(d t / 2 \pi)$-closure of $R(\bar{\Delta})$ and by the classical $\mathrm{F}$. and M. Riesz theorem (see Hoffman [18, p. 52]), it follows that $f \circ \varphi\left(e^{i t}\right) \neq 0$ a.e. on $\partial \Delta$. Hence $f(z) \neq 0$ a.e. $[m]$.

The following lemma is useful when trying to locate the spectrum of a subnormal operator if the spectrum of its minimal normal extension is known. For a proof, see Halmos [16, problems 157 and 158].

LEMMA (3.2). If $T$ is a subnormal operator and $N$ is its minimal normal extension, then $\sigma(N) \subset \sigma(T)$. Furthermore, if $G$ is a component of $C \backslash \sigma(N)$, then either $G \subset \sigma(T)$ or $G \cap \sigma(T)=\varnothing$.

We are now in a position to define $T$ and show that it possesses the desired properties. Let $A$ be a Cantor set of positive measure in the interval $[-1,1]$. Delete from $\bar{\Delta}$ the open disks whose diameters are the components of $[-1,1] \backslash A$ and let $X$ denote the resulting set. This set is usually referred to as the "string of beads". Let $U$ and $V$ be the components of int $X$ and fix points $a \in U$ and $b \in V$. Let $m_{a}$ be the representing measure for evaluation at $a$ on $R(\bar{U})$ and $m_{b}$ be the representing measure for evaluation at $b$ on $R(\bar{V})$.

If $m=m_{a}+m_{b}$ and $\mathcal{H}$ is the $L^{2}(m)$-closure of $R(X)$, then define $T$ : $\mathcal{H} \rightarrow \mathcal{H}$ by $(T f)(z)=z f(z)$. The operator $T$ is subnormal and its minimal normal extension is $M_{z}$, multiplication by $z$ on $L^{2}(m)$. Since $m_{a}$ and $m_{b}$ are mutually absolutely continuous with respect to arclength on $\partial U$ and $\partial V$, respectively, it follows that $\sigma\left(M_{z}\right)=\partial X$.

We now wish to show that $\sigma(T)=X$. If $\lambda \notin X$ then multiplication by $1 /(z-\lambda)$ defines an operator on $\mathcal{H}$ which is clearly the inverse of $T-\lambda I$. This implies that $\sigma(T) \subset X$. If $a$ and $b$ are in $\sigma(T)$, then it follows from Lemma (3.2) that $\sigma(T)=X$. Suppose $a \notin \sigma(T)$. Since $T-a I$ is invertible, there is an element $f \in \mathcal{H}$ such that $(T-a I) f(z)=(z-a) f(z)=1$ a.e. $[m]$. If $\left\{f_{n}\right\}$ is a sequence in $R(X)$ which converges to $f$ in $L^{2}(m)$, then $\left\{f_{n}\right\}$ also converges to $f$ in $L^{2}\left(m_{a}\right)$. Therefore,

$$
0=\int(z-a) f_{n} d m_{a} \rightarrow \int(z-a) f d m_{a}=\int 1 d m_{a}=1 .
$$

Since this is an obvious contradiction, we may conclude that $a \in \sigma(T)$. Replacing $a$ by $b$ in the above argument implies $b$ is also in $\sigma(T)$. Thus $X=\sigma(T)$ and since $T$ is subnormal, $X$ is a spectral set for $T$.

It remains to show that $T$ satisfies property (iii). Let $\mathscr{T}$ be a nontrivial invariant subspace for $T$ and suppose there exists $\omega \in U$ such that $\omega \notin$ $\sigma(T \mid \Re)$. Let $f$ be an arbitrary element of $\Re$. Since $(T-\omega I) \Re=\Re$, for each $n=1,2, \ldots$, there exists an element $f_{n} \in \mathfrak{N}$ such that $(z-\omega)^{n} f_{n}(z)$ $=f(z)$ a.e. $[m]$. We shall now consider the functions $f_{n}$ and $f$ as elements of $H^{1}\left(m_{a}\right)$. Each $h \in H^{1}\left(m_{a}\right)$ induces an analytic function $\tilde{h}$ on $U$ defined by $\tilde{h}(z)=\int h d m_{z}$. Since $(z-\omega)^{n} \tilde{f}_{n}(z)=\tilde{f}(z)$, it follows that $\tilde{f}$ has a zero of 
arbitrary order at $\omega$. Therefore, $\tilde{f}(z)=0$ for all $z \in U$, and hence, $f=0$ a.e. [ $\left.m_{a}\right]$. In particular, $f=0$ a.e. $\left[m_{a}\right]$ on the Cantor set $A$.

If we now consider $f$ as an element of $H^{1}\left(m_{b}\right)$, then it follows from (iii) of Lemma (3.1) that $f=0$ a.e. $\left[m_{b}\right]$. That is, $m_{b}(A)>0$ and $f=0$ a.e. $\left[m_{b}\right]$ on $A$. Therefore, $f=0$ a.e. $[m]$. But this implies that $\Re$ is trivial which gives a contradiction. Therefore $U \subset \sigma(T \mid \mathcal{T})$ and replacing $a$ by $b$ and $U$ by $V$, it also follows that $V \subset \sigma(T \mid \Re)$. Since we now have $X \subset \sigma(T \mid \Re), T$ must satisfy condition (iii).

4. Gleason parts. If $A$ is a function algebra on a compact set $X$, then $M_{A}$, the space of maximal ideals of $A$, can be identified with the nonzero multiplicative linear functionals defined on $A$. Since each element of $M_{A}$ has norm one, it follows that $\|\varphi-\psi\|<2$ for $\varphi, \psi \in M_{A}$. Furthermore, A. Gleason [15] discovered that $\|\varphi-\psi\|<2$ defines an equivalence relation on $M_{A}$ and the equivalence classes are referred to as the Gleason parts for $A$. If an equivalence class contains precisely one point, then it is called a trivial Gleason part. The following lemma gives a useful criterion for determining whether two elements $\varphi, \psi$ in $M_{A}$ are in the same Gleason part. Its proof, as well as the general theory of function algebras, may be found in the books by A. Browder [7] and T. Gamelin [13].

LEMMA (4.1). Two elements $\varphi$ and $\psi$ in $M_{A}$ are in different Gleason parts if and only if there exists a sequence $\left\{f_{n}\right\}$ of functions in $A$ such that $\left\|f_{n}\right\|<1$, $\varphi\left(f_{n}\right) \rightarrow 1$ and $\psi\left(f_{n}\right) \rightarrow 0$.

The maximal ideal space of $R(X)$ consists precisely of point evaluations on $X$ and, thus, may be identified with the set $X$. The Gleason parts for $R(X)$ then form a decomposition of $X$. D. Wilken [35] proved that each nontrivial Gleason part has positive 2-dimensional Lebesgue measure, which immediately implies that there is at most a countable number of nontrivial Gleason parts for $R(X)$. The trivial Gleason parts correspond to the peak points for $R(X)$. That is, those points $p$ for which there exists a function $f \in R(X)$ such that $f(p)=1$ and $|f(x)|<1$ for all $x \neq p$.

In [21], Mlak generalized Sarason's theorem by proving a decomposition theorem for operator-valued representations of arbitrary function algebras. The proof used function algebraic techniques and the decomposition was with respect to the Gleason parts. He later considered the representation of $R(X)$ into $\mathscr{B}(\mathcal{H})$ when $X$ is a spectral set for $T$ and gave a direct generalization of Theorem (2.4). See Theorem (4.2) below. Our intention is to show how this result can be used to produce reducing subspaces and also to locate the spectra of various operators. The proof of Mlak's theorem may be found in Mlak's paper [22] or the author's thesis [19]. We should also mention that 
Seever [28] has recently given a proof of Mlak's theorem using second dual techniques.

TheORem (4.2). Let $X$ be a spectral set for $T$ and let $G_{1}, G_{2}, \ldots$ be the nontrivial Gleason parts for $R(X)$. Then

$$
T=N \oplus\left(\sum_{i=1}^{\infty} \oplus T_{i}\right)
$$

where $N$ is normal with $\sigma(N) \subset \partial X$, and $\bar{G}_{i}$ is a spectral set for $T_{i}$ (in particular, $\left.\sigma\left(T_{i}\right) \subset \bar{G}_{i}\right)$.

Notice that von Neumann's theorem is a special case since by a theorem of Bishop [6], $R(X)=C(X)$ if and only if each point of $X$ is a peak point. Also Sarason's theorem follows from the fact that if $\mathbf{C} \backslash X$ has a finite number of components, then the nontrivial Gleason parts for $R(X)$ are precisely the components of int $X$ (see Ahern and Sarason [1] or Gamelin [13, p. 149]).

Recall that the operator $T$ given in $\S 3$ has the property that $\sigma(T)$ is a spectral set for $T$ and $T$ cannot be decomposed with respect to the two components of int $\sigma(T)$. In view of Theorem (4.2), this is not so surprising since even though int $\sigma(T)$ has more than one component, there is only one nontrivial Gleason part for $R(\sigma(T))$. This follows by observing that the two measures $m_{a}$ and $m_{b}$ are not mutually orthogonal.

D. Wilken [36] conjectured that if $G$ is a nontrivial Gleason part and $x \in \bar{G}$, then either $x \in G$ or $x$ is a peak point for $R(X)$. An affirmative answer to this conjecture was recently obtained by M. S. Melnikov [39]. Using Melnikov's result, we have the following corollary to Theorem (4.2).

CoRollary (4.3). If $X$ is a spectral set for $T$ and $\sigma(T)$ intersects at least two nontrivial Gleason parts for $R(X)$, then $T$ has a nontrivial reducing subspace.

Proof. Suppose $T$ does not have a nontrivial reducing subspace. The decomposition for $T$ in Theorem (4.2) is simply $T=T_{i}$ for some $i$. Now suppose a nontrivial Gleason part $G$ intersects $\sigma(T)$. It then follows that

$$
G \cap \sigma(T) \subset \sigma(T)=\sigma\left(T_{i}\right) \subset \bar{G}_{i},
$$

which implies $G \cap \bar{G}_{i} \neq \varnothing$. It now follows from Melnikov's result that $G$ must be $G_{i}$. Therefore, at most one nontrivial Gleason part can intersect $\sigma(T)$.

As previously mentioned, each $a \in X$ has a unique representing measure if $R(X)$ is a Dirichlet algebra. See [14] for conditions which imply that $R(X)$ is a Dirichlet algebra. One of the geometric conditions is that $\partial X$ is connected and the diameters of the components of $\mathbf{C} \backslash X$ are bounded away from zero. Using this, it is now easy to give examples in which $\mathbf{C} \backslash X$ has an infinite number of components and the nontrivial Gleason parts for $R(X)$ are precisely the components of int $(X)$. 
5. Peak sets for $R(X)$. In this section we use Theorem (4.2) to show how peak sets of $R(X)$ are related to reducing subspaces of $T$. A proper subset $M$ of $X$ is a peak set for $R(X)$ if there exists an $f \in R(X)$ such that $f(x)=1$ for all $x \in M$ and $|f(x)|<1$ for all $x \in X \backslash M$. If $M=\{a\}$ is a peak set for $R(X)$, then $a$ is a peak point for $R(X)$. A theorem of Bishop states that $R(X)=C(X)$ if and only if every point of $X$ is a peak point for $R(X)$ (see Gamelin [13, p. 54]).

TheOREM (5.1). If $X$ is a spectral set for $T$ and $M$ is a peak set for $R(X)$, then $T=R \oplus S$ with $\sigma(R) \subset M$ and $\sigma(S) \subset \overline{X \backslash M}$. Furthermore, $M$ and $\overline{X \backslash M}$ are spectral sets for $R$ and $S$, respectively.

Proof. Let $f$ be a function in $R(X)$ which is identically 1 on $M$ and whose modulus is strictly less than 1 off $M$. If $a \in M$ and $b \in X \backslash M$, then $f^{n}(a) \rightarrow 1$ and $f^{n}(b) \rightarrow 0$ as $n \rightarrow \infty$. Thus, by Lemma (4.1), $a$ and $b$ are in different Gleason parts. If $G_{1}, G_{2}, \ldots$ are the nontrivial Gleason parts for $R(X)$, then each $G_{i}$ is contained entirely in $M$ or entirely in $X \backslash M$. Let $A=\left\{i: G_{i} \subset M\right\}$ and $B=\left\{i: G_{i} \subset X \backslash M\right\}$.

By Theorem (4.2), $T=N \oplus\left(\sum_{i=1}^{\infty} \oplus T_{i}\right)$ where $N$ is normal with $\sigma(N) \subset$ $\partial X$ and $\bar{G}_{i}$ is a spectral set for $T_{i}$. It follows from Lemma (2.5) that $M$ is a spectral set for $\Sigma_{i \in A} \oplus T_{i}$ and $\overline{X \backslash M}$ is a spectral set for $\Sigma_{i \in B} \oplus T_{i}$. Since $N$ is normal, it may be decomposed as the direct sum of two normal operators $N_{1}$ and $N_{2}$ such that $\sigma\left(N_{1}\right) \subset M$ and $\sigma\left(N_{2}\right) \subset \overline{X \backslash M}$. If

$$
R=N_{1} \oplus\left(\sum_{i \in A} \oplus T_{i}\right) \text { and } S=N_{2} \oplus\left(\sum_{i \in B} \oplus T_{i}\right) \text {, }
$$

then $R$ and $S$ have the desired properties.

Corollary (5.2). Let $X=\sigma(T)$ be a spectral set for $T$ and let $M$ be a peak set for $R(X)$. If $M \varnothing \overline{X \backslash M}$, then $T$ has a nontrivial reducing subspace.

Proof. Let $R$ and $S$ be as in the previous theorem. Since $\sigma(T)=\sigma(R) \cup$ $\sigma(S)$, it is impossible for $T$ to equal $R$ or $S$. Hence both $R$ and $S$ are nontrivial operators.

Recall that an operator $T$ is completely nonnormal if $T$ has no reducing subspaces $\mathscr{T}$ such that $T \mid \mathfrak{T}$ is normal. Clancey and Putnam [9] have characterized the compact sets which are the spectra of completely nonnormal operators whose spectrum is a spectral set. In particular, they proved that if $X=\sigma(T)$ is a spectral set for $T$ and there exists an open disk $D$ which intersects $K$ with the property that $R(X \cap \bar{D})=C(X \cap \bar{D})$, then $T=R \oplus$ $S$ where $R$ is a normal operator. We shall show that this result follows from Theorem (4.2) and that it is possible to choose $R$ so that $\sigma(R)=\overline{X \cap D}$. The first part of the proof is similar to the proof given by Clancey and Putnam since both rely on the following theorem. 
TheOREM (5.3) (BISHOP's Localization THEOREM). If $f$ is continuous on $X$ and each $z \in X$ has a neighborhood $N(z)$ such that $f \in R(X \cap \overline{N(x)})$, then $f \in R(X)$.

For a proof, see Gamelin [13, p. 51].

Theorem (5.4). Let $X=\sigma(T)$ be a spectral set for $T$. If there exists an open disk $D$ such that $X \cap D \neq \varnothing$ and $R(\overline{X \cap D})=C(\overline{X \cap D})$, then $T=R \oplus S$ where $R$ is normal, $\sigma(R)=\overline{X \cap D}$, and $\sigma(S) \subset X \backslash D$.

Proof. We first observe that if $\lambda \in X \cap D$, then $\lambda$ is a peak point for $R(X)$. To show this, choose $\epsilon$ so that $B(\lambda ; 2 \epsilon)$, the open disk about $\lambda$ of radius $2 \epsilon$, is contained in $D$. Now construct a continuous function $f$ on $X$ such that $f(\lambda)=1, f$ is identically 0 on $X \cap B(\lambda ; \epsilon)$ and $|f(x)|<1$ for all $x$ in $X$ not equal to $\lambda$. It is easy to see that the hypotheses of Theorem (5.3) are satisfied. Hence, $f \in R(X)$ and, thus, $\lambda$ is a peak point for $R(X)$.

If $G_{1}, G_{2}, \ldots$ are the nontrivial Gleason parts for $R(X)$ and $\lambda \in X \cap D$, then it follows that $\lambda \notin \overline{\cup_{i=1}^{\infty} G_{i}}$. Let $T=N \oplus\left(\sum_{i=1}^{\infty} \oplus T_{i}\right)$ be the decomposition in Theorem (4.2). Since $\sigma\left(\sum_{i=1}^{\infty} \oplus T_{i}\right) \subset \cup_{i=1}^{\infty} G_{i}$, we must have $\overline{X \cap D} \subset \sigma(N)$. If $E$ is the spectral measure for $N$, then $R=N E(X \cap D)$ is normal, $\sigma(R) \subset \overline{X \cap D}$, and, since $D$ is open, $X \cap D \subset \sigma(R)$. Therefore, if

$$
S=N E(X \backslash D) \oplus\left(\sum_{i=1}^{\infty} \oplus T_{i}\right),
$$

then $T=R \oplus S, \sigma(R)=\overline{X \cap D}$ and $\sigma(S) \subset X \backslash D$.

6. Operators with thin spectra. If $X$ is the "string of beads" example discussed in $\$ 3$ and if the linear measure of the intersection of $X$ and the $x$-axis is zero, then the two components of the interior of $X$ belong to different Gleason parts for $R(X)$. This, in fact, is a special case of the more general result: if $X$ is compact, if $T$ is a piecewise $C^{2}$ closed Jordan curve which intersects $X$ in a set of measure zero on $\Gamma$, and $a$ and $b$ are in different components of the complement of $\Gamma$, then $a$ and $b$ are in different Gleason parts for $R(X)$. In fact, $X$ intersect the closure of the unbounded component in a peak set for $R(X)$. This result will provide sufficient conditions in terms of the spectrum for a subnormal operator to have a nontrivial reducing subspace.

We need the concept of analytically negligible in order to prove the above result. A set $E$ is analytically negligible if each function which is continuous on $\mathbf{C}$ and analytic on an open set $U$ can be approximated uniformly on $\mathbf{C}$ by functions which are continuous on $\mathbf{C}$ and analytic on $U \cup E$. Examples of analytically negligible sets and the relationship between analytic negligibility and continuous analytic capacity may be found in Gamelin [13, Chapter VIII]. Vitushkin [31] has shown that a piecewise $C^{2}$ curve is analytically 
negligible. Davie and Oksendal have proved the following theorem which relates analytic negligibility and rational approximation.

Theorem (6.1) (DAVIE AND OKSENDAL [10]). Let $K$ and $M$ be compact sets such that $\partial K \cap \partial M$ is analytically negligible. If $f \in C(K \cup M),\left.f\right|_{K} \in R(K)$, and $\left.f\right|_{M} \in R(M)$, then $f \in R(K \cup M)$.

If $\Gamma$ is a closed Jordan curve, then the bounded and unbounded components of the complement of $\Gamma$ will be denoted by $\Gamma_{1}$ and $\Gamma_{2}$, respectively.

TheOREM (6.2). If $X$ is compact and $\Gamma$ is a piecewise $C^{2}$ closed Jordan curve which intersects $X$ in a set of measure zero on $\Gamma$, then $X \cap \bar{\Gamma}_{2}$ is a peak set for $R(X)$. In particular, points in $X \cap \Gamma_{1}$ and $X \cap \bar{\Gamma}_{2}$ will be in different Gleason parts.

Proof. Let $M=\bar{\Gamma}_{1} \cap X$ and $K=\bar{\Gamma}_{2} \cap X$. Since the measure of $X \cap \Gamma$ is zero, it follows from a theorem of Rudin (see Hoffman [18, p. 8]), that there exists a continuous function $g$ defined on $\bar{\Gamma}_{1}$ such that $g$ is analytic on $\Gamma_{1}$, $g(z)=1$ for $z \in X \cap \Gamma$, and $|g(z)|<1$ for $z \notin X \cap \Gamma$. If we define $f$ on $X$ by $f(z)=g(z)$ for $z \in M$ and $f(z)=1$ for $z \in K$, then $f \in C(X),\left.f\right|_{M}=g \in$ $R(M)$, and $\left.f\right|_{K} \in R(K)$. Furthermore, $\partial K \cap \partial M$ is a subset of a piecewise $C^{2}$ curve and, hence, is analytically negligible. It now follows from Theorem (6.1) that $f \in R(X)$. Thus, $K$ is a peak set for $R(X)$.

TheOREM (6.3). Let $X$ be a spectral set for $T$. If $\Gamma$ is a piecewise $C^{2}$ Jordan curve which intersects $X$ in a set of measure zero on $\Gamma$, then $T=R \oplus S$ with $\sigma(R) \subset X \cap \bar{\Gamma}_{2}$ and $\sigma(S) \subset \overline{X \cap \Gamma_{1}}$.

Proof. Follows directly from Theorem (6.1) and Theorem (5.1).

COROllary (6.4). Let $T$ be a subnormal operator. If $\Gamma$ is a piecewise $C^{2}$ Jordan curve which intersects $\sigma(T)$ in a set of measure zero on $\Gamma, \sigma(T) \cap \Gamma_{1} \neq$ $\varnothing$, and $\sigma(T) \cap \Gamma_{2} \neq \varnothing$, then $T$ has a nontrivial reducing subspace.

Proof. By the above theorem, $T=R \oplus S$ with $\sigma(R) \subset \sigma(T) \cap \bar{\Gamma}_{2}$ and $\sigma(S) \subset \overline{\sigma(T) \cap \Gamma_{1}}$. Since $\sigma(T) \cap \Gamma_{i} \neq \varnothing, i=1,2$, it is impossible for either $R$ or $S$ to be trivial. Therefore, $T$ has a nontrivial reducing subspace.

Remarks. C. R. Putnam has obtained results similar to ours for subnormal operators. His approach differs from ours in that he uses the spectral measure of the minimal normal extension and also employs a "projection" operator technique. For more details, see the papers [24]-[26].

I would like to thank the referee for pointing out that Scott Brown [40] recently solved the invariant subspace problem for subnormal operators. His result has been generalized by Jim Agler [41] to operators whose spectra are spectral sets. 


\section{REFERENCES}

1. P. R. Ahern and D. Sarason, On some hypo-Dirichlet algebras of analytic functions, Amer. J. Math. 89 (1967), 932-941.

2. W. Arveson, Subalgebras of $C^{*}$-algebras. II, Acta Math. 128 (1972), 271-308.

3. S. K. Berberian, A note on operators whose spectrum is a spectral set, Acta Sci. Math. (Szeged) 27 (1966), 201-203.

4. C. A. Berger, A strange dilation theorem, Notices Amer. Math. Soc. 12 (1965), 590.

5. $\ldots$ A brief note on the existence of spectral sets, preprint.

6. E. Bishop, A minimal boundary for function algebras, Pacific J. Math. 9 (1959), 629-642.

7. A. Browder, Introduction to function algebras, Benjamin, New York, 1969.

8. K. F. Clancey, Examples of non-normal seminormal operators whose spectra are not spectral sets, Proc. Amer. Math. Soc. 24 (1970), 797-800.

9. K. F. Clancey and C. R. Putnam, Normal parts of certain operators, J. Math. Soc. Japan 24 (1972), 198-203.

10. A. M. Davie and B. K. Oksendal, Rational approximation on the union of sets, Proc. Amer. Math. Soc. 29 (1971), 581-584.

11. P. L. Duren, Theory of $H^{p}$ spaces, Academic Press, New York, 1970.

12. C. Foias, Some applications of spectral sets. I, Harmonic-spectral measure, Acad. R. P. Romine Stud. Cerc. Mat. 10 (1959), 365-401; English transl., Amer. Math. Soc. Transl. (2) 61 (1967), 25-62.

13. T. W. Gamelin, Uniform algebras, Prentice-Hall, Englewood Cliffs, N. J., 1969.

14. T. W. Gamelin and J. Garnett, Pointwise bounded approximation and Dirichlet algebras, J. Functional Analysis 8 (1971), 360-404.

15. A. Gleason, Function algebras in Seminar on Analytic functions. Vol. II, Institute for Advanced Study, Princeton, N. J., 1957, pp. 213-226.

16. P. R. Halmos, A Hilbert space problem book, Van Nostrand, Princeton, N. J., 1967.

17. E. Hewitt and K. Stromberg, Real and abstract analysis, Springer, New York, 1965.

18. K. Hoffman, Banach spaces of analytic functions, Prentice-Hall, Englewood Cliffs, N. J., 1962.

19. R. G. Lautzenheiser, Spectral sets, reducing subspaces, and function algebras, $\mathrm{Ph}$. D. Thesis, Indiana University, 1973.

20. A. Lebow, On von Neumann's theory of spectral sets, J. Math. Anal. Appl. 7 (1963), 64-90.

21. W. Mlak, Decompositions and extensions of operator valued representations of function algebras, Acta Sci. Math. (Szeged) 30 (1969), 181-193.

22. __ Partitions of spectral sets, Ann. Polon. Math. 25 (1972), 273-280.

23. C. R. Putnam, The spectra of subnormal operators, Proc. Amer. Math. Soc. 28 (1971), 473-477.

24. Invariant subspaces of certain subnormal operators, J. Functional Analysis 17 (1974), 263-273.

25. , Generalized projections and reducible subnormal operators, Duke Math. J. 43 (1976), 101-108.

26. __ Peak sets and subnormal operators, Illinois J. Math. 21 (1977), 388-394.

27. D. Sarason, On spectral sets having connected complement, Acta Sci. Math. (Szeged) 26 (1965), 289-299.

28. G. L. Seever, Operator representations of uniform algebras. I, preprint.

29. J. G. Stampfli, A local spectra theory for operators. IV. Invariant subspaces, Indiana Univ. Math. J. 22 (1972), 159-167.

30. M. Tsuji, Potential theory, Maruzen, Tokyo, 1959.

31. A. G. Vitushkin, Analytic capacity of sets and problems in approximation theory, Uspehi Mat. Nauk 22 (1967), 141-199; Russian Math. Surveys 22 (1967), 139-200.

32. J. von Neumann, Eine Spektraltheorie für allgemeine Operatoren eines unitären Raumes, Math. Nachr. 4 (1951), 258-281.

33. B. L. Wadhwa, A hyponormal operator whose spectrum is not a spectral set, Proc. Amer. Math. Soc. 38 (1973), 83-85. 
34. J. Wermer, Dirichlet algebras, Duke Math. J. 27 (1960), 373-382.

35. D. Wilken, Lebesgue measure for parts of $R(X)$, Proc. Amer. Math. Soc. 18 (1967), 508-512. 36. The support of representing measures for $R(X)$, Pacific J. Math. 26 (1968), 621-626.

37. J. P. Williams, Minimal spectral sets of compact operators, Acta Sci. Math. (Szeged) 28 (1967), 93-106.

38. L. Zalcman, Analytic capacity and rational approximation, Lecture Notes in Math., vol. 50, Springer-Verlag, Berlin, 1968.

39. M. S. Melnikov, On the Gleason parts of the algebra $R(X)$, Mat. Sb. 101 (143) (1976), No. 2 (10), 293-300. (Russian)

40. S. W. Brown, Some invariant subspaces for subnormal operators, preprint.

41. Jim Agler, An invariant subspace theorem, preprint.

Department of Mathematics, Rose-Hulman Institute of Technology, Terre haute, INDIANA 47803 